\title{
Tuberculosis incidence in Cameroonian prisons: A 1-year prospective study
}

\author{
J Noeske, ${ }^{1} \mathrm{MD}, \mathrm{PhD} ; \mathbf{N}$ Ndi, ${ }^{2} \mathrm{MD} ;$ G Amougou Elo, ${ }^{3} \mathrm{MD} ; \mathbf{S}$ Mbondi Mfondih $^{4}$ \\ ${ }^{1}$ Independent Senior Consultant, National Tuberculosis Programme, Yaounde, Cameroon \\ ${ }^{2}$ Penitentiary Administration, Ministry of Justice, Yaounde, Cameroon \\ ${ }^{3}$ Regional Delegation of Prison Administration, N'Gaoundere, Cameroon \\ ${ }^{4}$ Health/AIDS Programme, German International Cooperation, Yaounde, Cameroon
}

Corresponding author: J Noeske (juergennoeske@yahoo.fr)

Background. Rates of tuberculosis (TB) transmission in prisons are reported to be high worldwide. However, a recent systematic review identified only 19 published studies reporting TB incidence in prisons, most of them from the last century and only one from subSaharan Africa.

Objectives. To assess the persisting risk of smear-positive pulmonary tuberculosis (PTB) among prison populations benefiting from a comprehensive TB/HIV control programme in Cameroon, compared with that in the community.

Methods. This descriptive and prospective study evaluated PTB incidence rates over a 1-year period. The study population was inmates of 10 major prisons, sampled by convenience, comprising about $45 \%$ of the country's prison population. As PTB incident cases, all prisoners with incident PTB after a prison stay of $\geq 90$ days were considered. The prison TB incidence rate was compared with that of the corresponding male population in the community.

Results. The mean annual PTB incidence in Cameroonian prisons in this study was 1700 cases in 100000 person-years at risk, the incidence rate ratio being 9.4 (95\% confidence interval 8.1 - 10.9).

Conclusion. Findings suggest that internationally recommended prison TB control measures alone may not help protect prisoners from within-prison spread of TB. Imprisonment policies and conditions therefore require fundamental changes.

S Afr Med J 2014;104(3):209-211. DOI:10.7196/SAMJ.7384

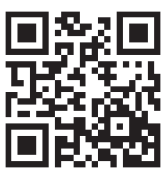

Rates of tuberculosis (TB) transmission in prisons are reported to be high worldwide. ${ }^{[1]}$ Owing to the constant movement of prisoners to the community and vice versa, prisons are a recognised reservoir for TB transmission to the community. However, the magnitude of this public health problem remains largely unknown, particularly in sub-Saharan Africa (SSA). ${ }^{[2]}$ While it is generally accepted that the most appropriate epidemiological measure for quantifying TB morbidity is incidence rates, a recent systematic review identified only 19 published studies reporting TB incidence in prisons, most of them from the last century and only one from SSA. ${ }^{[3,4]}$

In Cameroon, TB and HIV control measures have been implemented as a pilot intervention over the past several years in 10 major urban, semi-urban and rural prisons by the Ministry of Justice
(MoJ), with technical and financial assistance from a development agency. The 10 target prisons are situated in different regions of the country, and hold about $45 \%$ of the total prison population of 23500 All 10 prisons were built in the 1960s and are therefore outdated. They are characterised by severe overcrowding, the overall mean overpopulation being 200\% according to the MoJ, but reaching $>1000 \%$ in some cells and cell blocks. The official budget amounts to about US $\$ 0.45$ per inmate per day. Medical services are substandard. The prison population consists of both pre-trial and sentenced detainees and is characterised by a large annual turnover $(>70 \%)$, yet the average duration of stay exceeds 3 months for the majority of cases ( $>90 \%)$. About $85 \%$ of new prison entrants accept voluntary counselling and testing for HIV infection. The mean HIV infection rate among inmates dropped from $5.8 \%$ in 2011 to $5.0 \%$ in 2012 , 
following a national trend, but is still almost double that of the comparable civilian population (2.9\% in men in 2011, according to the third Demographic and Health Survey $\left.{ }^{[5]}\right)$. An average of three times more female inmates than males are infected $(15.3 \%$ v. $5.5 \%$ in 2011, according to the unpublished annual activity report of the MoJ). TB control activities follow the guidelines and use the management tools of the National TB Control Programme (NTP). Case finding according to NTP guidelines is passive. In the 10 target prisons, however, three active case finding strategies have been added as routine procedure: medical screening at entry with identification of suspects based on a clinical score, contact tracing in the prison, and periodic (at least annual) mass screening in four larger and heavily overcrowded prisons where TB transmission has been shown to remain high in spite of the control measures implemented. ${ }^{[5]}$

This study sought to assess the risk of smear-positive pulmonary tuberculosis (PTB) morbidity among prison populations in Cameroon compared with that in the corresponding community.

\section{Methods}

This was a descriptive and prospective study that collected PTB incidence data and calculated incidence rates over a 1-year period (October 2011 - September 2012). The study population comprised only inmates of the 10 prisons targeted by the HIV/TB Control Programme of the MoJ; prison healthcare and other staff were not considered. For each prison, data on prisoners diagnosed with PTB during the study period, as well as the date of their admission to prison, were extracted from the standardised TB patient registers (World Health Organization (WHO) format, adapted). Only microbiologically confirmed TB cases were taken into account. Information on the prison population was retrieved monthly (on the first day of each month) from the prison's admission registers. As PTB disease incident cases, all prisoners with incident PTB after a length of stay of $>90$ days were considered; cases diagnosed with PTB during the first 3 months after entry were considered to have been prevalent cases at entry ${ }^{[3]}$ To determine the person-time of inmates at risk in any of the target prisons, the monthly count of inmates per prison was added up. The prison population in Cameroon is $>96 \%$ male, aged between 15 and 54 years with a median age of about 30 years. To determine PTB incidence among the comparable general population, the NTP's notification data for the male population aged between 15 and 54 years in the towns (for larger urban prisons) or administrative districts (for semi-urban or rural prisons) where the prisons were situated were used. Population figures were calculated using the 2005 national census, estimating an annual population growth of $2.6 \%{ }^{[7]}$

All data on PTB incidence in the prisons were captured in an EpiData entry database (version 3.1), mimicking an adapted version of the standard (WHO format) TB treatment register. Data on TB incidence in the communities were collected on an MS Excel sheet. Analyses were performed showing 95\% confidence intervals (CIs) for proportions and $p$-values where appropriate.

Written permission to conduct the study was obtained from the NTP. Ethical clearance was given by the Cameroon National Ethics Committee.

\section{Results}

Table 1 presents findings for each prison studied and for all 10 prisons together. Overall, for 10468 person-years of risk, $178 \mathrm{~TB}$ cases were notified, or 1700 cases per 100000 person-years of follow-up. During the same period, the incidence (notification) of TB among the general population was 178 per 100000 person-years of follow-up. The incidence rate ratio (IRR) was 9.4 (95\% CI 8.1 - 10.9).

\section{Discussion}

TB prevalence studies in Cameroonian prisons during recent years have shown widely varying results. ${ }^{[6,8]}$ This is the first systematic TB incidence study covering almost half of the country's prison population in a variety of prisons. All 10 prisons included in the study were benefiting from a comprehensive TB and HIV control programme implemented several years previously and designed and executed according to international recommendations. Despite this, the mean PTB case notification rate among inmates remained almost 10 times higher (IRR 9.5) than the corresponding national average. The study findings showed that the burden of PTB varied from prison to prison. This may be due to various factors, including local differences in conditions of incarceration, in particular overcrowding and lack of ventilation; regional differences in TB and HIV incidence and prevalence; regional differences in rates of poverty; and local differences in access to quality prison health services.

The results of this study are consistent with those of Baussano et al. ${ }^{[4]}$ who reported a median estimated annual incidence of TB of 1943 per 100000 persons for prisons in middle- and low-income countries. However, the reported median estimated IRR for TB

Table 1. Tuberculosis incidence in prisons and communities in Cameroon, 2011 - 2012

\begin{tabular}{|c|c|c|c|c|c|}
\hline \multirow[b]{2}{*}{ Prison } & \multirow[b]{2}{*}{ Cases (at risk), $n$} & \multicolumn{2}{|c|}{ Incidence $(/ 100000)$} & \multirow[b]{2}{*}{ Incidence rate difference } & \multirow[b]{2}{*}{ IRR (95\% CI) } \\
\hline & & Prisons & General population & & \\
\hline CP Yaounde & $63(3791)$ & 1662 & 138 & 1524 & $11.9(9.2-15.7)$ \\
\hline CP Douala & $42(2900)$ & 1448 & 200 & 1248 & $7.2(5.3-9.7)$ \\
\hline CP Ebolowa & $4(380)$ & 1053 & 262 & 793 & $4.0(1.5-10.9)$ \\
\hline CP Maroua & $30(900)$ & 3333 & 297 & 3036 & $10.9(7.5-15.9)$ \\
\hline CP Bafoussam & 14 (999) & 1401 & 190 & 1211 & $7.3(4.2-12.5)$ \\
\hline PP Foumban & $7(256)$ & 2734 & 212 & 2522 & $12.6(5.8-27.3)$ \\
\hline PP Edea & $7(284)$ & 2465 & 219 & 2246 & $11.0(5.2-23.4)$ \\
\hline PP Nkongsamba & $3(365)$ & 822 & 239 & 583 & $3.4(1.1-10.8)$ \\
\hline PP Mbanga & $7(279)$ & 2509 & 481 & 2028 & $5.1(2.4-11.1)$ \\
\hline PP Mora & $1(314)$ & 318 & 48 & 270 & $6.6(0.9-48.6)$ \\
\hline Total, $N$ & $178(10468)$ & 1700 & 178 & 1522 & $9.4(8.1-10.9)$ \\
\hline
\end{tabular}


of 32.8 (interquartile range (IQR) 15.4 - 36.1) was higher. This difference may be because the studies cited by Baussano et al. were done in the 1990s and/or in settings where TB outbreaks were not yet driven by the HIV epidemic. In SSA, figures for Ivory Coast alone reported a TB incidence rate in the penal camp of Bouake prison of 5 803/100 000 population and an IRR of 32.8 (IQR 27.1 - 39.6). This study also dates from the early 1990s (1990 - 1992), before the implementation of a national TB control programme aimed at collecting reliable case notification figures in the community and providing access to effective treatment.

Our findings also illustrate what Basu et al. ${ }^{\left[{ }^{[9]}\right.}$ when modelling TB transmission in prisons, referred to as 'institutional amplifiers', demonstrating that even a substantial increase in case detection and treatment success rates alongside traditional control measures has little effect on the overall population incidence, as long as such hubs of transmission continue to exist. Likewise, Uys et al. ${ }^{[10]}$ when calculating TB transmission possibilities in low- and high-prevalence areas and settings, concluded that in high-prevalence settings transmission is relatively unresponsive to changes in the number of infectious people. Uys et al. coined the term 'transmission elasticity' to describe this phenomenon and warn against excessively optimistic projections regarding the effectiveness of 'habitual' control strategies. According to these authors, even if conventional control measures remain the priority, key interventions to reduce TB incidence, prevalence and mortality should include limiting the number of persons entering prisons, decreasing overcrowding, implementing environmental measures such as improved ventilation, and ensuring contact tracing.

HIV/TB co-infection data are reported by the NTP without gender and age group breakdown. This study was therefore unable to compare HIV/TB co-infection rates among inmates with those in the comparable general population. However, according to our observations the role of HIV infection as a risk factor for PTB is significantly less important in prisons than among the general population: HIV/TB co-infection rates reported for 2011 were $11.2 \%$ among inmates with TB and $31.3 \%$ among the general population (NTP annual report 2011, unpublished). In the Cameroonian context, characterised by a generalised HIV epidemic and a considerable burden of TB due to HIV, this could be taken as an argument for the specific impact of confinement conditions on TB transmission. ${ }^{[6]}$

Extrapolation of the results to the remaining prisons in Cameroon may underestimate $\mathrm{TB}$ incidence rates, considering that all 10 of the prisons studied have benefited from a comprehensive TB control programme.

In terms of its strengths, this study gathered data prospectively and applied a recommended strategy for measuring morbidity in Cameroonian prisons. However, it was based on routinely collected data, which are only as accurate as the data collection system permits. The study had the following limitations: (i) the study population comprised only about $45 \%$ of Cameroon's total prison population, which affected the representativeness of the results; (ii) person-time of observation was estimated by adding up the monthly count of inmates, which affected the accuracy of the incidence figures obtained; and (iii) the 15 - 20\% of inmates undergoing their first 3 months of incarceration were not subtracted from the total person-time of observation, possibly contributing to an under-estimation of incidence rates. ${ }^{[3]}$ The prison population targeted in this study remained relatively constant during the past 5 years with regard to monthly turnover rates and age and gender distribution. Adapting the denominator by excluding those $20 \%$ of inmates who could be assumed to be in the first 3 months of incarceration at any time would increase the incidence of PTB in prisons to 2126 per 100000 person-years, and the IRR would rise to 11.9 (IQR 10.3 - 13.8).

\section{Conclusion}

Our findings suggest that internationally recommended prison TB control measures alone may not adequately protect prisoners from within-prison spread of TB and thereby reduce the national TB burden. Rather, imprisonment policies and conditions require fundamental changes. ${ }^{[6,11]}$

Author contributions. JN, NN, GAE and SMM planned the manuscript, GAE, NN and SMM collected the data, JN, NN, GAE and SMM analysed the data, JN drafted the first version of the manuscript, and all authors contributed towards critically revising the manuscript. All authors read and approved the final manuscript.

\section{References}

1. World Health Organization. Literature review on TB control in prisons. http://who.int//tb/challenges/tp/ prisons/tb_in_prisons_lit_review_10Fev2008 (accessed 15 July 2013).

2. O'Grady J, Hoelscher M, Atun R, et al. Tuberculosis in prisons in sub-Saharan Africa - the need for improved health services, surveillance and control. Tuberculosis (Edinb) 2011;91(2):173-178. [http://dx.doi. org/10.1016/j.tube.2010.12.002]

3. Rieder HL, Anderson C, Dara M, et al. Methodological issues in quantifying the magnitude of the tuberculosis problem in a prison population. Int J Tuberc Lung Dis 2011;15(5):662-667.

Baussano I, Williams BG, Nunn P, Beggiato M, Fedeli U, Scano F. Tuberculosis incidence in prisons: A

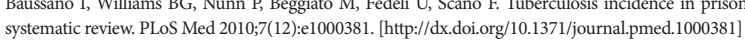
systematic review. PLoS Med 2010; $7(12): e 1000381$. [http:///dx.doi.org/10.1371/journal.pmed.1000381]
Institut National de la Statistique, Ministère de l'Économie de la Planification et de l'Aménagement du . Institut National de la Statistique, Ministère de P'Économie de la Planification et de l'Aménagement du
Territoire Ministère de la Santé Publique. 2011 Cameroon Demographic and Health Survey and Multiple Indicators Cluster Survey (DHS-MICS). Yaounde 2012. http://www.statistics-cameroon.org (accessed 14 September 2013)

6. Noeske J, Ndi N, Mbondi MS. Controlling TB against imprisonment conditions - a lost case: Experience from Cameroon. Int J Tuberc Lung Dis 2011;15(2):223-227.

Bureau Central des Recensements et des Etudes des Populations. Rapport de présentation des résultats définitifs. Yaounde 2010. http://www.statistics-cameroon.org/news.php?id=18 (accessed 15 July 2013).

Noeske J, Kuaban C, Amougou G, Piubello A, Pouillot R. Pulmonary tuberculosis in the central prison of Douala, Cameroon. East Afr Med J 2006;83(1):25-30. [http://dx.doi.org/10.4314/eamj.v83i1.9357]

9. Basu S, Stuckler D, McKee M. Addressing institutional amplifiers in the dynamics and control of tuberculosis epidemics. Am J Trop Med Hyg 2011;84(1):30-37. [http://dx.doi.org/10.4269/ajtmh.2011.0-0472]

10. Uys P, Marais BJ, Johnston-Robertson S, Hargrove J, Wood R. Transmission elasticity in communities Uys P, Marais BJ, Johnston-Robertson S, Hargrove J, Wood R. Transmission elasticity in communities
hyperendemic for tuberculosis. Clin Infect Dis 2011;52(12):1399-1404. [http://dx.doi.org/10.1093/cid/ cir229]

11. Reid SE, Topp SM, Turnbull ER, et al. Tuberculosis and HIV control in sub-Saharan African prisons 'Thinking outside the prison cell'. J Infect Dis 2012;205(Suppl 2):S265-S273. [http://dx.doi.org/10.1093/ infdis/jis029]

Accepted 17 October 2013. 